BULLETIN OF THE

AMERICAN MATHEMATICAL SOCIETY

Volume 77, Number 3, May 1971

\title{
A NOTE ON NONRIGID NASH STRUCTURES
}

BY D. R. J. CHILLINGWORTH AND J. HUBBARD

Communicated by Michael Artin, September 3, 1970

1. Introduction. Geometry in the Nash category is intermediate between real algebraic and real analytic geometry, and provides a link whereby methods of algebraic geometry can be applied to some problems in differential topology. See [1] for example.

The Nash structure of a manifold embedded in $R^{n}$ is not deformed by perturbations of the embedding; this is a consequence of the uniqueness theorem of Nash [2]. In the context of the general problem of deformation of Nash structures, Mazur has asked whether these "embedding" structures are in fact rigid (see below for definitions). We show here that for the unit circle $S^{1}$ this is not the case.

We wish to thank Barry Mazur for introducing us to Nash manifolds, and we are most grateful to him for the several conversations in which he showed us how to present our own examples.

2. Definitions. A real analytic function $f: U \rightarrow R$ on an open set $U \subset R^{n}$ is called algebraic if there is a nontrivial real polynomial $P$ such that $P\left(x_{1}, \cdots, x_{n}, f\right)=0$ where the $x_{i}$ are the coordinate functions. A Nash manifold is a topological space $M$ together with a sheaf $\mathcal{O}_{M}$ of local rings over $M$ such that every point $p \in M$ has a neighbourhood $V$ on which $\theta_{M} \mid V$ is isomorphic to the sheaf $\Theta_{U}$ of germs of algebraic functions on open subsets of an open subset $U$ of $R^{n}$. Alternatively, a Nash manifold can usefully be thought of as a real analytic manifold with maximal atlas $\left\{U_{\alpha}, \phi_{\alpha}\right\}$ of charts such that the overlap maps $\phi_{\beta} \circ \phi_{\alpha}^{-1}: \phi_{\alpha}\left(U_{\alpha} \cap U_{\beta}\right) \rightarrow \phi_{\beta}\left(U_{\alpha} \cap U_{\beta}\right)$ are all coordinate-wise algebraic. A real analytic map $\psi: M \rightarrow N$ is a Nash map if $\psi^{*} \Theta_{N} \subset \Theta_{M}$. Nash manifolds $M, N$ are equivalent $(M \cong N)$ if there is a diffeomorphism $h: M \rightarrow N$ such that $h$ and $h^{-1}$ are Nash maps. A Nash manifold $M$ is rigid if any real analytically locally trivial Nash map $p: E \rightarrow B$ with $p^{-1}(b) \cong M$ for some $b \in B$ is locally trivial (in the Nash sense) at $b$.

EXAMPLES. (1) $R^{n}$ has a canonical Nash structure $R_{c}^{n}$; we take $U=R^{n}$ in the above.

(2) A real algebraic embedding $i: M \rightarrow R^{n}$ induces an embedding structure $M_{i}$ on $M$ from $R_{c}^{n}$ by $\mathcal{O}_{M i}=i * \Theta_{R^{n}}{ }_{i}$. This structure does not depend on the choice of embedding [2] so we denote it by $M_{\text {emb }}$.

AMS 1970 subject classifications. Primary 32C05, 32G99; Secondary 13H99, 16A58, 18F20.

Key words and phrases. Nash manifold, real algebraic manifold, rigid. 
(The "real algebraic manifold" structures in [2] are precisely these embedding structures.)

(3) The covering map $e: R \rightarrow S^{1}: x \mapsto \exp 2 \pi i x$ induces a Nash structure $S_{\exp }^{1}$ on $S^{1}$ from $\boldsymbol{R}_{c}$. The Nash manifolds $S_{\mathrm{emb}}^{1}$ and $S_{\exp }^{1}$ are not equivalent, since $S_{\mathrm{emb}}^{1}$ admits (global) nonconstant Nash maps $S_{\mathrm{emb}}^{1} \rightarrow R_{c}$ (e.g. $(x, y) \mapsto x$, taking $\left.S^{1} \subset R^{2}\right)$ whereas $S_{\exp }^{1}$ does not; any Nash map $S_{\exp }^{1} \rightarrow R_{c}$ lifts to an algebraic function $R \rightarrow R$ which is periodic and therefore constant.

3. The structures $S_{\alpha}^{1}$. Consider a Nash structure $\boldsymbol{R}_{\sin }$ defined on $R$ as follows: on any open set $V \subset R$ the Nash functions (i.e. the elements of $\left.\mathcal{O}_{R_{s i n}} \mid V\right)$ are precisely those real analytic functions algebraically dependent on $\sin 2 \pi x$. In the neighbourhood of any point, $\sin 2 \pi x$ or $\cos 2 \pi x$ will define a chart for this structure. Note that $e: R \rightarrow S^{1}$ is a Nash map $\boldsymbol{R}_{\mathrm{sin}} \rightarrow S_{\mathrm{emb}}^{1}$, and $S_{\mathrm{emb}}^{1}=\boldsymbol{R}_{\sin } / \boldsymbol{Z}$. Note also that under addition $R_{\sin }$ is a group object in the Nash category, since $\sin 2 \pi(x+y)$ is algebraically related to $\sin 2 \pi x$ and $\sin 2 \pi y$ (by the well-known formula!).

Let $E=R_{\sin }^{+} \times R_{\sin }$, regarded as a group over $R_{\sin }^{+}=\left\{x \in R_{\sin } \mid x>0\right\}$. Translation by sections of the subgroup $F=\{(x, y) \in E \mid y / x \in \boldsymbol{Z}\}$ gives a free action of $\boldsymbol{Z}$ on $E$, and by iteration of the sine formula it is seen to be a Nash action. This defines an exact sequence

$$
0 \rightarrow F \rightarrow E \rightarrow E / F \rightarrow 0
$$

of Nash groups over $R_{\sin }^{+}$. The real analytic diffeomorphism $E \rightarrow E$ : $(x, y) \mapsto(x, y x)$ takes $F$ onto $\boldsymbol{R}_{\sin }^{+} \times \boldsymbol{Z}$ and hence induces a real analytic isomorphism between the above sequence and

$$
0 \rightarrow Z \rightarrow R \rightarrow R / Z \rightarrow 0
$$

understood as an exact sequence of trivial bundles over $R_{\text {sin }}^{+}$. Therefore $\bar{E}=E / F$ is real analytically equivalent to $R^{+} \times R / Z=R^{+} \times S^{1}$.

Let $p: R^{+} \times S^{1} \rightarrow R^{+}$denote projection on the first factor, and let $S_{\alpha}^{1}$ denote $p^{-1}(\alpha)$ with the Nash structure induced by that of $\bar{E}$. Observe that $S_{1}^{1}=S_{\text {emb }}^{1}$.

Proposition. $p: \bar{E} \rightarrow R_{\sin }^{+}$is not locally trivial as a Nash map. In particular, $S_{\alpha}^{1} \cong S_{\beta}^{1}$ if and only if $\alpha / \beta$ is rational; thus $S_{1}^{1}$ is not rigid.

Proof. The (non-Nash) map

$$
\gamma: E \rightarrow E:(x, y) \mapsto(\beta x / \alpha, \beta y / \alpha)
$$

preserves $F$ and hence induces a map $\bar{\gamma}: \bar{E} \rightarrow \bar{E}$. The restriction $\bar{\gamma} \mid p^{-1}(\alpha): S_{\alpha}^{1} \rightarrow S_{\beta}^{1}$ is easily checked to be a Nash isomorphism when $\alpha / \beta$ is rational, $\sin 2 \pi \alpha y$ being algebraically related to $\sin 2 \pi \beta y$. 
We now show that when $\alpha / \beta$ is irrational there exists no Nash map $S_{\alpha}^{1} \rightarrow S_{\beta}^{1}$ of nonzero degree, which proves $S_{\alpha}^{1} \not S_{\beta}^{1}$. Such a map $\phi: S_{\alpha}^{1} \rightarrow S_{\beta}^{1}$ of degree $k$ lifts to $\tilde{\phi}: R \rightarrow R$ satisfying $\tilde{\phi}(x+\alpha)=\tilde{\phi}(x)+k \boldsymbol{\beta}$ and a nontrivial polynomial relationship $P(\sin 2 \pi x, \sin 2 \pi \tilde{\phi}(x))=0$. For fixed $x$ the number of values mod 1 of $\tilde{\phi}(x+n)(n \in Z)$ is finite, so there are $m(x), n(x) \in \boldsymbol{Z}$ such that $\tilde{\phi}(x+n)=\tilde{\phi}(x)+m$. For most $x$ this holds with $m(x), n(x)$ constant in a neighbourhood of $x$, hence it holds everywhere with $m, n$ constant since $\tilde{\phi}$ is analytic. Letting $\phi_{1}(x)=\tilde{\phi}(x)-(m / n) x$, we have $\phi_{1}(x+n)=\phi_{1}(x)$ and $\phi_{1}(x+\alpha)=\phi_{1}(x)$ $+(k \beta-m \alpha / n)$, which imply $\alpha / \beta=k n / m$.

(Note. Our original proof applied only when $\beta=1$. We are indebted to $\mathrm{P}$. Deligne for this improved version.)

Remark 1. Assume now $\alpha$ irrational. Since $\phi_{1}$ has periods 1 and $\alpha$ it must be constant, so up to rotation there exists precisely one Nash map $S_{\alpha}^{1} \rightarrow S_{\beta}^{1}(\alpha / \beta$ rational) of each degree $k \neq 0$. If $k=0$ then $m=0$, and there exist no nonconstant Nash maps $S_{\alpha}^{1} \rightarrow S_{\beta}^{1}$ of degree zero for any $\beta$. A similar proof shows there exist no nonconstant Nash maps $S_{\alpha}^{1} \rightarrow S_{\text {exp }}^{1}$, or $S_{\alpha}^{1} \rightarrow R_{c}$.

REMARK 2. A Nash $Z$-action on $E$ given by $n(x, y)=\left(2^{n} x, 2^{n} y\right)$ preserves $F$ and induces an action on $\bar{E}$ which takes fibres to fibres. The quotient space $\bar{E} / \boldsymbol{Z}$ is a torus equipped with a Nash structure such that the Nash map $p^{\prime}: \bar{E} / \boldsymbol{Z} \rightarrow \boldsymbol{R}_{\mathrm{sin}}^{+} / \boldsymbol{Z}=S_{\mathrm{emb}}^{1}$ induced from $p$ is a trivial real analytic fibration over $S^{1}$ but is not locally trivial in the Nash sense.

REMARK 3. Barry Mazur has shown us a general $n$-dimensional setting for the above. Take $\boldsymbol{R}_{\sin }^{n}=\boldsymbol{R}_{\mathrm{sin}} \times \boldsymbol{R}_{\mathrm{sin}} \times \cdots \times \boldsymbol{R}_{\mathrm{sin}}$ ( $n$ times), and let $B$ denote the space of all bases of the $R$-vector space $R_{\text {sin }}^{n}$. Since $B$ is an open subset of $R_{\sin }^{n^{2}}$ it inherits a Nash structure. Let $E=B \times R_{\text {sin }}^{n}$ regarded as a Nash group over $B$, and let $F \subset E$ be the subgroup defined by $F_{b}=$ discrete subgroup of $R_{\sin }^{n}$ generated by $b$ $(b \in B)$. Taking the quotient $E / F$ we obtain a family $\left\{T_{b}\right\}_{b \in B}$ of $n$ tori over $B$, and it can be shown by an extension of the above arguments that $T_{b}, T_{b^{\prime}}$ are equivalent if and only if $b$ and $b^{\prime}$ lie in the same orbit under the natural action of $\operatorname{GL}(n, Q)$ on $B$.

\section{REFERENCES}

1. M. Artin and B. Mazur, On periodic points, Ann. of Math. (2) 81 (1965), 82-99. MR 31 \#754.

2. J. F. Nash, Jr., Real algebraic manifolds, Ann. of Math. (2) 56 (1952), 405-421. MR 14, 403.

Institut des Hautes Études Scientifiques, 91-Bures-sur-Yvette, France

Faculté des Sciences, 91-Orsay, France 